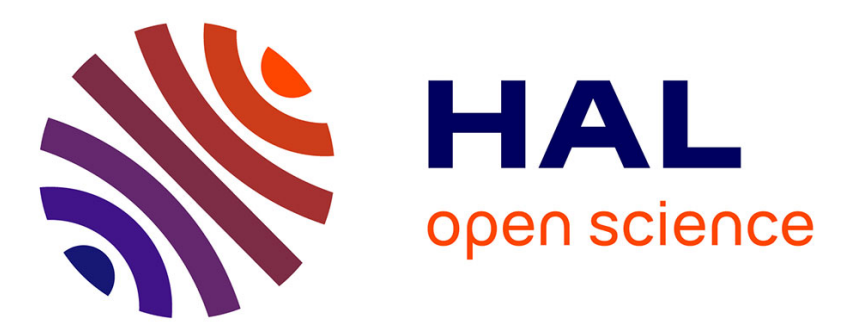

\title{
Plant membrane-protein mediated intracellular traffic of fatty acids and acyl lipids
}

\author{
Yonghua Li-Beisson, Jens Neunzig, Youngsook Lee, Katrin Philippar
}

\section{To cite this version:}

Yonghua Li-Beisson, Jens Neunzig, Youngsook Lee, Katrin Philippar. Plant membrane-protein mediated intracellular traffic of fatty acids and acyl lipids. Current Opinion in Plant Biology, 2017, 40, pp.138-146. 10.1016/j.pbi.2017.09.006 . hal-01709038

\section{HAL Id: hal-01709038 \\ https://hal-amu.archives-ouvertes.fr/hal-01709038}

Submitted on 14 Feb 2018

HAL is a multi-disciplinary open access archive for the deposit and dissemination of scientific research documents, whether they are published or not. The documents may come from teaching and research institutions in France or abroad, or from public or private research centers.
L'archive ouverte pluridisciplinaire HAL, est destinée au dépôt et à la diffusion de documents scientifiques de niveau recherche, publiés ou non, émanant des établissements d'enseignement et de recherche français ou étrangers, des laboratoires publics ou privés. 


\section{Plant membrane-protein mediated intracellular traffic of fatty acids and acyl lipids}

YonghuaLi-Beisson $^{1}$, JensNeunzig $^{2}$, YoungsookLee ${ }^{3}$, KatrinPhilippar $^{2}$

1 CEA, CNRS and Aix-Marseille Universite' , Institut de Biosciences et Biotechnologies d'Aix-Marseille, UMR 7265, CEA Cadarache, SaintPaul-lez Durance F-13108, France

2 Saarland University, Center for Human- and Molecular Biology - Plant Biology, Campus A 2.4, D-66123 Saarbru“" cken, Germany

3 Division of Integrative Bioscience and Biotechnology, POSTECH, Pohang 37673, Republic of Korea Corresponding author: Philippar, Katrin (katrin.philippar@uni-saarland.de)

In plants, de novo synthesis of fatty acids (FAs) occurs in plastids, whereas assembly and modification of acyl lipids is accomplished in the endoplasmic reticulum (ER) and plastids as well as in mitochondria. Subsequently, lipophilic compounds are distributed within the cell and delivered to their destination site. Thus, constant acyl-exchanges between subcellular compartments exist. These can occur via several modes of transport and plant membrane-intrinsic proteins for FA/lipid transfer have been shown to play an essential role in delivery and distribution. Lately, substantial progress has been made in identification and characterization of transport proteins for lipid compounds in plant organelle membranes. In this review, we focus on our current understanding of protein mediated lipid traffic between organelles of land plants.

\section{Introduction}

Fatty acids (FAs) are building blocks for acyl lipids, which in the form of phospho-lipids or galactolipids are assembled into membranes or as triacylglycerol (TAG) oils represent a major form of carbon storage in plant cells. FAs and lipids, excreted into the apoplast form the lipophilic barriers made of waxes, cutin, suberin or sporopollenin that are essential for protection against water loss and pathogen attack or necessary for pollen fertility [1]. Senescence, cold temperatures and nutrient starvation require FA/lipid remodeling and turnover to sequester carbon energies, protect against freezing or to replace phospholipids by plastid-derived galactolipids, respectively [2-4]. Further, FA breakdown in peroxisomes plays an essential role in the maintenance of lipid homeostasis as well as during germination. Thus, proper maintenance and regulation of lipid metabolism is not only crucial for membrane function but also essential for plant development, growth and performance. Plant lipid metabolism therefore has been subjected to intensive studies and found to involve hundreds of enzymatic reactions, occurring in several subcellular compartments [5,6]. In turn, this requires exchange of FAs, lipids and their metabolic intermediates between organelles and membrane systems. Because lipophilic molecules can't move freely in an aqueous cellular environment and their transport has to be directional, several modes of intracellular lipid traffic exist. These include membrane contact sites, diffusion within the same membrane system, vesicular trafficking and direct transport of molecules. Substantial progress has been made in identifying membraneintrinsic proteins, which either actively transport FA/lipid compounds or mediate their intracellular 
exchange. In this review, we highlight recent advances in characterization of these proteins and the current knowledge on FA/lipid traffic between organelles. Excretion of lipophilic compounds across the plasma membrane via ATP-binding cassette $(\mathrm{ABC})$ transporters is summarized in [1]. For non membrane-associated processes, lipid transfer and acyl-CoA binding proteins, membrane contact sites, vesicle traffic, or acyl-exchange mechanisms, we point to recent reviews [7-11]. However, it is noteworthy, that for FA/lipid traffic in general most likely membrane contacts as well as tight cooperation between transport and biosynthesis steps are required. The flow of FAs and lipids into cellular metabolism often drives their transport across membranes, for example, during plastid FA export where membrane-transporter mediated facilitated diffusion of FAs most likely is driven by the activation of free FAs to acyl-CoA and their subsequent incorporation into acyl lipids.

\section{Transport between plastids and ER}

Since all long-chain FAs are made in plastids and mainly assembled into acyl lipids either in plastids or in the ER, and because ER-derived lipids are components of plastid intrinsic membranes, extensive FA/lipid transport between these two organelles is required. De novo FA synthesis occurs in the plastid stroma where acyl chains grow while attached to the acyl carrier protein (ACP) $[5,12]$. Part of these FAs is integrated into lipids inside plastids (prokaryotic pathway), leading to the formation of chloroplast intrinsic lipids such as monogalactosyl-diacylglycerol (MGDG), digalactosyl-diacylglycerol (DGDG), sulfoquinovosyl-diacylglycerol (SQDG) and phosphatidylglycerol (PG). These lipids mainly constitute the plastid intrinsic thylakoid and inner envelope (IE) membrane systems and contain a large fraction of $\mathrm{C}_{16: 3}$ acyl chains, since the plant plastid sn2acyltransferase exclusively uses $\mathrm{C}_{16}$-FA substrates [13]. The majority of plastid-made FAs, however, is exported to the ER for further elongation, acyl editing and lipid assembly via the eukaryotic pathway. Here, the phospholipids phosphatidyl-choline (PC), phosphatidyl-ethanolamine (PE), and phosphatidyl-inositol(PI) as well as TAG oils and precursors for complex extracellular lipophilic compounds such as wax, cutin, suberin and sporopollenin are produced. In the eukaryotic pathway, the ER sn2-acyltransferase has a high specificity for $\mathrm{C}_{18}$-FAs. Depending on plant species, a considerable amount of these eukaryotic lipids can also be transferred into plastids for lipid assembly [14]. As a consequence, a eukaryotic-type acyl-glycerol backbone has to be imported into plastids [15].

\section{Plastid FA export to the ER}

In the plastid stroma, the three carbon compound pyruvate is metabolized by pyruvate dehydrogenase to form acetyl-CoA, the starting two carbon unit for de novo FA synthesis. Whereas pyruvate can be made inside plastids, it is also the product of glycolytic sucrose breakdown in the cytosol. Thus, it has long been supposed that transporters in the plastid envelope membranes could play an essential role in the uptake of pyruvate from the cytosol for FA synthesis, export and subsequent acyl lipid production. The contribution of pyruvate transporter in plastid envelopes (Figure 1) to TAG synthesis in the ER has recently been demonstrated by seed-specific overexpression of the $\mathrm{Na}^{+}$-dependent pyruvate transporter BASS2 (bile acid:sodium symporterfamily protein 2; [16]) in Arabidopsis thaliana [17 ${ }^{\circ}$. In the BASS2 over-expressor lines, the seed oil content 
was increased by $\approx 27 \%$, highlighting that for successful modulation of lipid metabolism, transport as well as synthesis steps have to be targeted. So although BASS2 does not transport lipophilic compounds itself, its activity increases the flow of FAs from plastids to the ER by providing precursors to FA synthesis.

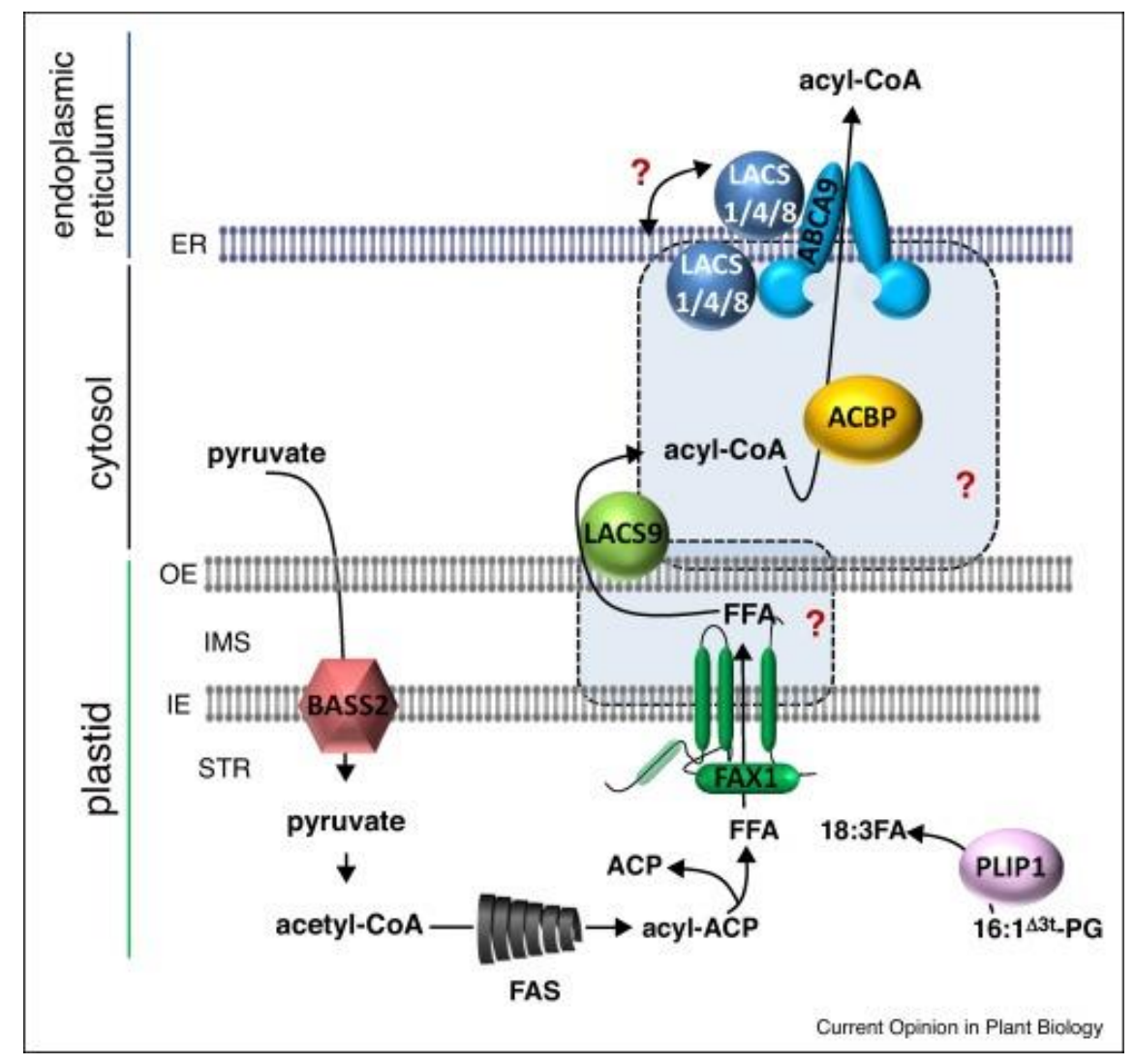

Figure 1. Plastid FA export to the ER. In addition to plastid-intrinsic pyruvate, the C3 organic acid can be imported from the cytosol via the BASS2 protein (bile acid:sodium symporter family protein 2). For BASS2, a chloroplast targeting signal and about eight membrane-spanning helices are predicted (Aramemnon database [16,55]). Although not explicitly shown [16], BASS2 therefore most likely integrates into the inner envelope membrane (IE). In the plastid stroma(STR) pyruvate is converted to acetyl-CoA and thereby fed into the fatty acid synthesis machinery (FAS). After release from the acyl carrier protein (ACP) export of free fatty acids (FFA) across the IE is mediated by FAX1 [21*]. FAX1 most likely comprises three membrane-spanning and one amphiphilic helix, as well as an N-terminal $\alpha$-helical domain and is proposed to transport FFAs in a passive carrier-like mode, driven by a strong gradient of FFAs from the stroma-side of the IE to the cytosolic face of the outer envelope membrane (OE; see [22] for transport mechanism). Here the long-chain acyl CoA synthetase LACS9, that has been shown to be attached to the cytosolic face of the OE [56], in addition might activate FA export by producingacyl-CoA. Since acyl-CoA is either further metabolized, imported into the ER or captured by acyl-CoA binding proteins (ACBP), a constant flow of plastid FAs into metabolism can be provided. Thereby a gradient of newly synthesized FAs from the plastid stroma to the cytosol is generated, which besides the ATP-dependent activation by LACS enzymes could represent a driving force for plastid FA export. Besides de novo synthesis, the phospholipase PLIP1 might provide free FA substrates for FAX1 (mainly $C_{18: 3} F A$ ) by hydrolyzing plastid specific 16:1 $1^{43 t}-P G\left[18^{*}\right]$. The mechanism of the transport of FAs across the intermembrane space (IMS) and the $O E$ as well as if OE/IE membrane contacts are involved remains unclear. LACS 1, 4 and 8 enzymes are localized to the ER. If they are active at the cytosolic 
face or lumen, however, is not elucidated. Since LACS 1, 4 and 8 seem to be able to compensate loss of function of LACS9, at the cytosolic ER side they might participate in activation of FA export from plastids. In general LACS enzymes are not described to contain membrane spanning domains, however, according to the plant membrane protein database Aramemnon [55], one $\alpha$ helical transmembrane domain is weakly predicted. Whether the traffic of acyl-CoA through the cytosol is mediated via proteins such as ACBP [10], or occurs through ER-plastid contact sites is not unraveled yet. Finally, transport into the ER occurs through a dimer of the half-size ABC transporter ABCA9. Each ABCA9 monomer consists of a permease domain with six transmembrane helices and a nucleotide binding domain (see [27,28] for overview of ABC transporter). For ABCA9, transport of the substrate acyl-CoA is postulated [29], however, transport of FFA and co-operation with LACS1, 4, 8 at the ER lumen might be possible as well. The latter transport mode has been demonstrated for FA import into peroxisomes via the full-size ABC transporter ABCD1 [57]. ABCD1 cleaves cytosolic acyl-CoA, transports FFAs, and interacts with the enzymes LACS6 and/or LACS7 that activate FFAs to acyl-CoA in the lumen of the peroxisome (see [58]). Whether ABCD1 releases CoA to the cytosol or peroxisome lumen, however, is still unknown as well. Possible involvement of membrane contacts between OE/IE and OE/ER are depicted as pale blue boxes.

Once synthesized, acyl residues are either integrated into lipids via plastid-resident acyltransferases, or released as free FAs by hydrolysis via acyl-ACP thioesterases at the stroma-side of the plastid inner envelope (IE) $[5,12]$. Only very recently the action of the plastid intrinsic phospholipase PLIP1 was proposed to initiate a mechanism leading to plastid FA export [18*0]. By specifically releasing polyunsaturated acyl groups (mainly $\mathrm{C}_{18: 3}$ ) from the $s n l$ glyceryl position of a unique plastid PG (i.e. 16:1 $1^{\Delta 3 \mathrm{t}}$-PG), PLIP1 would thereby provide free FAs for export from plastids and feeding into TAG synthesis in embryos of developing seeds [18*0. Free FAs, however, are toxic and therefore a quick flow into metabolism or export from the organelle is required. In general it is agreed that free FAs are shuttled across plastid envelopes, however, it was a matter of debate whether this occurs by simple diffusion or if membrane transport proteins are involved [19]. A facilitated diffusion of free FAs through the lipid membrane was supported by the finding that an acylACP synthetase (SynAas) in the cyanobacterium Synechocystisis sufficient for membrane FA transfer [20]. Here the authors provide a model in which free FAs can cross a membrane bilayer by first integrating into the membrane according to their physicochemical properties and subsequent retrieval from the membrane phase by the ATP-dependent activation to acyl-ACP via the action of SynAas. Thus, the driving force for FA membrane transport is activation and feeding into metabolism.

Insight into a transporter mediated mechanism of plastid FA-export in land plants was provided by the identification of Arabidopsis FAX1 (fatty acid export 1), which inserts into the chloroplast IE by $\alpha$-helicalmembrane-spanning domains (Figure $1\left[21^{*}\right]$ ). FAX1 function is crucial for biomass production, male fertility and distribution of FA-derived compounds such as lipids, ketone waxes or pollen cell wall materials. ER-derived lipids decreased when FAX1 was absent, but levels of plastid-produced species increased. FAX1 over-expressing lines showed the opposite phenotypes, including a rise of TAGs in flowers and leaves. In yeast FAX1 further complements for FA transport. Thus, in IE membranes FAX1 could function in a process called vectorial acylation represented by a passive, carrier-like transport mechanism. This FAX1-mediated facilitated diffusion most likely is 
driven by a strong gradient of free FAs fed into lipid metabolism at the cytosolic face of the plastid envelope (for details of transport mechanisms, see [22]).

The long-chain acyl-CoA synthetase LACS9 at the outer envelope (OE) is unique in plastids, catalyzes about $90 \%$ of the plastid activity for acyl-CoA synthesis [23,24] and thus via its ATPdependent, thioestercoupling of FAs to CoA might drive vectorial acylation for plastid FA-export (Figure 1). Because lacs 9 knockouts show wild-type phenotype, and so far no additional plastid LACS was identified, an overlapping function of LACS9 could be provided by the ER-targeted LACS1, LACS4 and LACS8 $\left[25^{*}, 26\right]$. Whereas lacs $1 /$ lacs 9 and lacs4/lacs 9 double mutants show reduced FA content in seed TAGs, the mutation of LACS 8 in the lacs4/lacs 9 mutant background is lethal. The observation that the absence of the plastid envelope-localized LACS9 can be compensated by other LACS enzymes (i.e. LACS1,4,8) suggest that activity of these enzymes should be present at the cytosolic face of the membrane (Figure 1). The proteinaceous domains for membrane attachment and the precise localization of LACS enzyme activity towards the ER lumen, cytosol or at plastid-ER contact sites, however, are still unclear. Moreover, LACS4 and LACS9 might also be implicated in plastid lipid import (see below), indicating tight co-operation between plastid and ER localized proteins. These scenarios suggest that depending on the metabolic situation and driving forces, the same proteins can contribute to exchange of lipid compounds between organelles in both directions.

In the cytosol, acyl-CoA can be captured by acyl-CoA binding proteins (ACBPs, for overview see [10]), which provide a shuttle for the subcellular distribution of acyl chains but also supply a sink for FAs and thereby might contribute to drive plastid FA-export. Finally, ABCA9, a halfsize Arabidopsis ABC transporter of subfamily A (for overview see [27,28]), was described to be involved in FA/acyl-CoA import into the ER in seeds (Figure 1 [29]). Here, the driving force for transport is ATP-hydrolysis, directly by the ATP-binding cassette domains of the ABC transporter. ABCA9 is localized to ER membranes and is expressed in seeds at storage lipid accumulation stages. The TAG amount as well as weight and size are reduced in abca 9 knockout seeds but increased in ABCA9-overexpressors. Feeding experiments with ${ }^{14} \mathrm{C}$-labeled oleoyl-CoA and oleic acid demonstrated reduced FA incorporation in TAGs of abca 9 seeds, revealing a key role for ABCA9 during FA/acyl-CoA import into the ER.

Besides the action of membrane transporters such as FAX1 (IE) and ABCA9 (ER) as well as LACS enzymes at both compartments, it is discussed that plastid to ER transfer of lipid compounds can occur via membrane contact sites. In principle, it seems likely that both membrane contacts and membrane transport are necessary for efficient transfer of lipid compounds [8]. Moreover, the phospholipid PC and the more water soluble metabolic intermediates lyso-PC and acyl-CoA via the so-called Lands cycle, could also play a role in lipid transfer from plastids to the ER [8].

\section{Lipid import from the ER into plastids}

In Arabidopsis about 50\% DAG-backbone precursors of prokaryotic and eukaryotic pathways are used for assembly of plastid galactolipids. Thus, lipid import from the ER into plastids is substantial. At the chloroplast IE, a prokaryote-type ABC transporter system made of TGD1, TGD2 and TGD3 (named 'trigalactosyl-diacylglycerol 1-3' after the oligogalactolipids that accumulate upon their 
mutation) carries out this function [7,15]. TGD1 represents the IE membraneintrinsic permease [30,31], TGD2 binds the substrate in the intermembrane space, and the stromalocalized ATPase TGD3 provides the energy for the transport (Figure $2[32,33]$ ). At the OE the $\beta$ barrel lipid transfer protein TGD4 was described to be involved in lipid import [34,35]. Since TGD2 as well as TGD4 have a PA binding capacity, most likely this phospholipid represents the transport intermediate for the TGD translocon complex. The fifth TGD component is TGD5, a small glycinerich protein that was identified in a screen for lipid trafficking mutants [36" $]$. Based on genetic and co-immunoprecipitation analyses, TGD5 was suggested to bridge the intermembrane space by binding to TGD4 and the TGD1-3 complex and thereby facilitating lipid handover from the OE to the IE (Figure 2). Plastid galactolipid synthesis occurring at the OE and IE membrane systems is then fueled by PA, either imported from the ER or produced inside the plastid. Interestingly, PA is able to interact with MGDG synthase MGD1 at the IE as well as with the N-terminal region of DGDG synthase DGD1 at the OE (Figure 2). On the one hand, the PA-MGD1 interaction, which most likely is of hydrophobic nature, leads to an increased MGDG production, indicating an important role of PA in the modulation of galactolipid synthesis [37]. The activation of MGD1 together with the conversion of PA into plastid intrinsic lipids might thus represent an additional driving force for lipid uptake. On the other hand, PA-binding by the N-terminal region of DGD1 enables OE/IE membrane association as well as transfer of lipids between both membranes [38 $]$, thereby further stimulating the flow of lipids into synthesis pathways, in this case DGDG production.

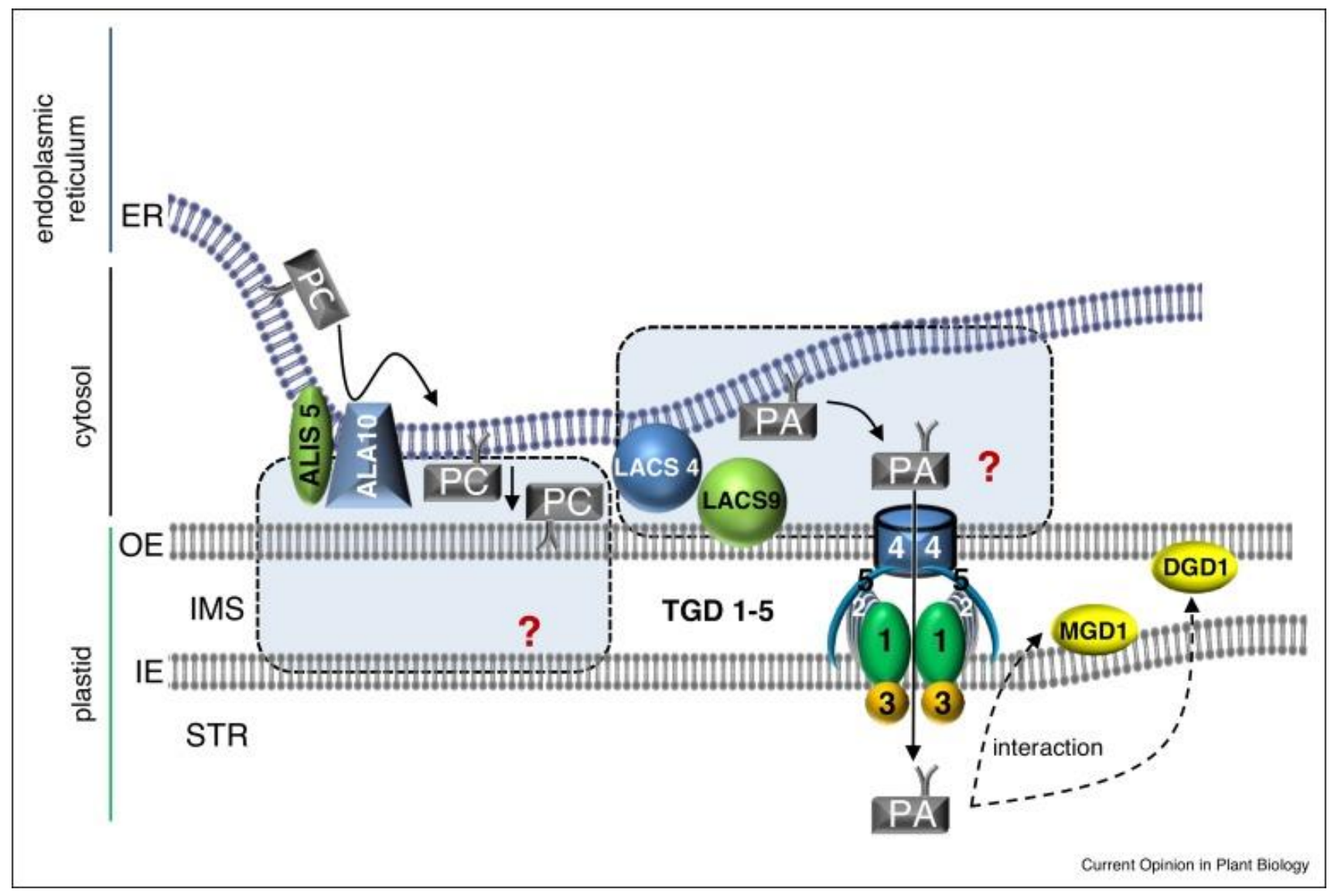

Figure 2. Lipid import from the ER into plastids. Import of eukaryotic lipids into plastids is accomplished via a prokaryotic-type ABC transporter system composed of the subunits TGD1, 2 and 3 at the inner envelope membrane (IE). Since TGD subunits have been shown to bind to phosphatidic 
acid (PA), this phosphoglycerolipid might represent the transport substrate. However, it has not been proven yet what the TGD complex transports and other lipids might be possible substrates as well. In contrast to the half-size ABC transporter ABCA9 (see Figure 1), the TGD 1-3 complex is assembled by separate polypeptidesubunits. The six transmembrane-domain permease subunit TGD1 inserts as dimer into the IE and binds the two nucleotide-binding domains TGD3 at the stroma (STR) face. Similar to prokaryotic ABC transporters (see [27]), TGD2 represents the substrate, that is, PAbinding domain in the intermembrane space(IMS) and is linked to the IE with one $\alpha$-helix (predicted by Aramemnon, [55]). For TGD2 an octameric composition in the complex is postulated [59]. At the plastid outer envelope membrane (OE) PA-binding is mediated by the $\beta$-barrelprotein TGD4. Interestingly TGD4 is predicted to assemble as homo dimer into the OE where each subunit contains four antiparallel $\beta$-sheets [35]. Connection of TGD4 at the OE and TGD1-3 at the IE might be mediated by the small glycine-rich TGD5, which is expected to bridge the intermembrane space (IMS). For TGD5, two potential $\alpha$-helical membrane domains (Aramemnon [55]) most likely insert into the IE (see model in [36“]). After import, eukaryotic PA is fed into plastid lipid synthesis. Specifically galactolipid synthesis occurring at the two envelope membranes is additionally stimulated by PA. First, PA has been reported to allostericallyactivate monogalactosyldiacylglycerol synthase(MGD1) at the IE, which potentially depicts an important mechanism for regulation of lipid synthesis [37]. Second, the binding of PA to the digalactosyl-diacylglycerol synthase (DGD1) at the inner leaflet of the OE has been described to enable OE/IE association as well as lipid transfer [38']. How eukaryotic PA is transferred from the ER to TGD4 remains unclear, however, ER-plastid contact sites are likely (pale blue box) and involvement of LACS4 at the ER as well as LACS9 at the OE has been postulated. Further, the concerted action of LACS4 and LACS9 is described to be involved in plastid import of ER-made phophatidylcholine (PC) as well (see [8,25*] for discussion). In the ER membrane PC is flipped to the outer leaflet by the action of the P4type ATPase(flippase) ALA10 that inserts into the ER membrane with ten $\alpha$-helical membrane spanning domains (see [41]). Interaction of ALA10 with the two-membrane domain protein ALIS5 in the ER seems to provide attachment to the plastid and thereby transfer of PC to the OE (see $\left.\left[41,42^{*}\right]\right)$. Interestingly, however, ALIS5 is predicted to contain a chloroplast targeting signal (Aramenon [55]). Thus, in future research it has to be clarified whether ALSI5 might be dually localized to ER and plastids and thereby manage targeting of ALA10 near plastids or if interacting proteins are involved. The required protein components for contact on the plastid side, however, are still unknown (indicated by pale blue box).

The relative contribution of the prokaryotic and eukaryotic pathway to plastid lipid synthesis varies depending on plant species. In 16:3 plants, considerable amount of plastid-intrinsic lipids are made of the prokaryotic pathway, whereas in 18:3 plants like Arabidopsis except for PG, which is thought to be still plastid-made, ER-derived lipids provide essentially all precursors for plastid lipid synthesis. This suggests different functioning and fine-tuning of the lipid import machineries, represented by the presumed orthologs of TGD complexes in microalgae and land plants. Lately, Yang et al. [39*0] demonstrated that a 27 amino acid loop of TGD1 that interacts with TGD2 has specifically adapted to the metabolic requirements of either 16:3 or 18:3 plant species, indicating co-evolution of lipid transport proteins and metabolism. Moreover, the lack of eukaryotic galactolipid species in chloroplasts of the green microalgae Chlamydomonas reinhardtiiindicates that here the protein mediated import of lipids to chloroplasts is probably not necessary. Again this metabolic situation is reflected by the set of TGD-complex proteins: whereas TGD1-3 in Chlamydomonasmost likely 
contribute to lipid traffic between chloroplast envelopes [40], TGD4 and TGD5 for import from the ER across the $\mathrm{OE}$ are absent in the Chlamydomonas proteome (see [22] for further discussion).

Since in vivo pulse-chase labeling experiments revealed that in leaves of lacs4/lacs 9 double mutants, ER-derived galactolipids were reduced in chloroplasts, Jessen et al. proposed a model in which LACS9, together with LACS4, is involved in FA-transfer from the ER to plastids (Figure 2[25" $]$ ). This transfer most likely occurs in the form of $\mathrm{C}_{18: 2}$ in eukaryotic PA or PC at plastid-ER contact sites $\left[8,25^{\circ}\right]$. The composition of these contact sites, that is, with high PC content, further supports transfer of PC, including lyso-PC and acyl-CoA via the Lands cycle (see [8]). Interestingly, while absent in the IE and thylakoids, PC is the main component of the plastid OE membrane [41] and therefore is very likely to be involved in transfer of lipids with extra-plastid compartments.

Only recently, the P4-type ATPase ALA10, which belongs to the family of phospholipid flippases, was described to play a role in PC transfer from the ER to the plastid [42*0. ALA10 interacts with the fatty acid desaturase FAD2 at the ER and thereby most likely flips eukaryotic PC to the cytosolic side of the ER membrane (energy by direct ATPconsumption of the ATPase). When interacting with ALIS5 (ALA-interacting subunit 5), ALA10 further can be targeted to near the surface of plastids (Figure 2). Upon its over-expression, ALA10 thereby is able to increase transfer of ER-made PC to chloroplasts, most likely at ER-plastid contact sites [42**].

In summary, PA - most likely via the TGD complex — and PC - via LACS and ALA10 action are likely to be the two major lipid classes shuttled from the ER to plastids. However, mathematical modeling favors DAG as the transport substrate [43]. Thus, the mode and substrates of ER to plastid lipid traffic still are a matter of discussion (see [8]).

\section{Lipid transport into mitochondria}

Although de novo FA synthesis occurs also in mitochondria, major components of mitochondrial membranes are made essentially of lipids synthesized by other organelles. Only for PG, the direct precursor of the mitochondria-specific cardiolipin, biosynthesis enzymes are associated with a localization in mitochondria (see [44]). The de novo mitochondria pathway is thus found to provide acyl precursors mostly for synthesis of lipoic acid, lipid A, and cardiolipins $\left[5,45,46^{\circ}, 47^{\circ}\right]$. To build their membranes, mitochondria therefore have to import most lipids from other compartments. Unlike in yeast where extensive lipid exchange of mitochondria with the ER and the vacuole has been demonstrated [48] knowledge about this inter-organellar lipid traffic in plant cells is scarce (see [44]). Recently, Michaud et al. [44] hypothesized that lipids could be made by enzymes present in the outer mitochondrial membrane (OM). Indeed the PECT1 (phospho-ethanolamine-cytidyl transferase), a key enzyme of PC and PE synthesis in plants has been shown to sit at the cytosolic face of the OM [49]. This would require contact sites between ER and mitochondria, which might be provided by the MELL1 protein (mitochondria-ER-localized LEA-related LysM domain protein 1). MELL1 was described in the moss Physcomitrella patens [50,51], and so far represents the only protein identified that might link mitochondria-ER contact sites in plants. 
Under phosphate limitation, however, it is well known that the plastidmade galactolipid DGDG compensates for the loss of phospholipids in cellular membranes including mitochondria [52,53]. Here, the number of plastid-mitochondria contacts increases, PE is exported from mitochondria for phosphate recycling in the cytosol whereas DGDG from plastids is imported into mitochondria (see [44]). Only recently a membrane biochemical approach identified the protein Mic60 at the inner membrane (IM) of plant mitochondria to play an important role in this lipid traffic (Figure $3\left[54^{\circ}\right]$ ). In parallel to findings in yeast, At-Mic60 together with the OM $\beta$-barrel protein TOM40, which is well known for forming the protein import pore, is an essential part of the so-called MICOS complex that connects the OM and IM in mitochondria. Further, At-Mic60 is part of a large lipid-binding complex (mitochondrial transmembrane lipoprotein, MTL), and mediates DGDG flow from plastids into mitochondria during phosphate starvation [44,54*]. The driving forces for this transport as well as other protein partners, however, so far remain unknown.

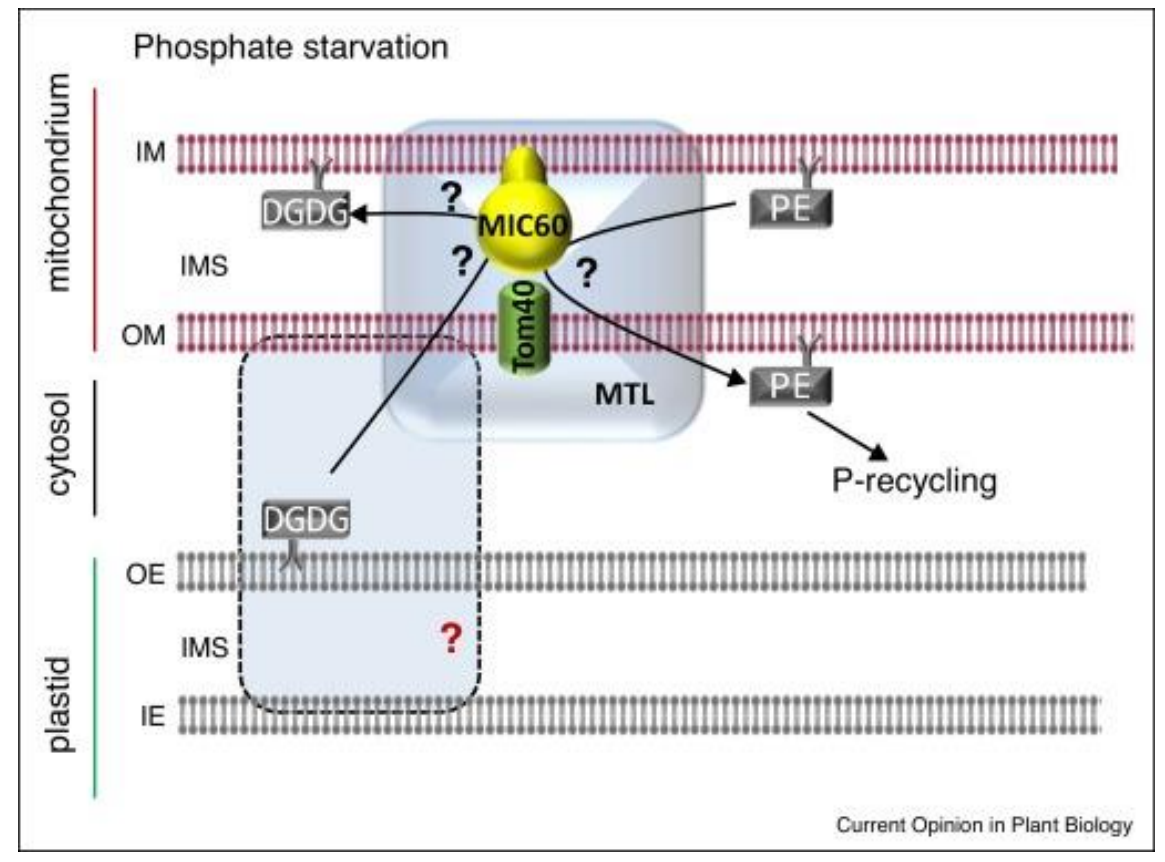

Figure 3. Lipid transfer between plastids and mitochondriaduring phosphate starvation. In Arabidopsis mitochondria, the protein MIC60 is most likely integrated with one $\alpha$ helical membrane domain into the inner membrane (IM; Aramemnon [54*,55]). In the so-called MICOS (mitochondria contact site cristaeorganizing system) complex, well known in yeast mitochondria [48], At-MIC60 was described to bridge the intermembrane space (IMS) together with proteins of the TOM complex (protein translocon at the outer membrane of mitochondria), such as the $\beta$-barrel protein TOM40 in the outer membrane (OM). Although the main function of TOM40 is protein import into mitochondria, it is further postulated to act together with MIC60 and several still unknown proteins (question marks) in a so-called mitochondrial transmembrane lipoproteincomplex (MTL; see [44,54*]). During phosphate-limiting conditions it was described that At-MIC60 within the MTL complex has a function in transfer of the membrane lipid phosphatidylethanolamine (PE) from the IM to the OM. PE is then further exported for degradation, thereby recycling a phosphate group for cellular metabolism and a diacylglycerol backbone for lipid synthesis. In turn, the plastidmade, bilayer-forming galactolipid digalactosyl-diacylglacerol (DGDG) is provided to mitochondrial membranes to substitute for the lost phospholipids. This DGDG lipid import again is mediated by MIC60 at the IM. Most likely this lipid exchange between plastids and mitochondria 
during phosphate starvation occurs via membrane contact sites (indicated by pale blue box). Protein components at the plastid envelope involved in this stress-induced lipid traffic, however, still await identification.

In summary, recent characterization of membrane transport proteinsinvolved in intracellular FA/lipid traffic has added substantial new and exciting information to unravel the process of plant lipid homeostasis and distribution. However, several open questions remain and still many proteins await identification as well as detailed molecular characterization.

Perspectives and open questions

In general in future research, it will be necessary to clarify correct membrane topology (i.e. number of membrane-spanning domains, orientation with respect to cytosol or lumenof organelles) as well as detailed subcellular localization of the proteins involved in FA/lipid transport in plant cells. Further, interaction partners as well as proteins mediating membrane contact between organelles have to be identified. Since in vivotargeting of fluorescence-tagged proteins due to over-expression of constructs quite often leads to mistargeting and even modulation of membrane systems in organelles (see [56]), the future challenge is to confirm and refine subcellular localizations and membrane subdomains via alternative techniques like membrane biochemistry or immuno-histochemical studies. For transport proteins, direct assays are required to elucidate transport activity and substrate specificities. In addition, it will be extremely helpful to integrate and combine the enormous knowledge on plant acyl lipid synthesis with the emerging function of FA/lipid transport proteins. Whereas energy for transport via ABC transporters or flip transfer by P4-type ATPAses is readily available by direct ATP-binding, the driving forces for the so-called vectorial acylation transport of FAs are provided by ATP-dependent activation of FAs to acyl-CoA, establishment of concentration gradients across membranes (compare Figure 1) and subsequent feeding into metabolism. Further, lipid binding of enzymes as demonstrated for PA-MDG1, PA-DGD1 interaction might further stimulate synthesis and thereby also transport of acyl lipid compounds. In the case of FA/lipid transport of mitochondria further proteins involved have to be identified to be able to speculate about driving forces.

During FA export from plastids (Figure 1) it is still unclear how FAs travel from FAX1 in the IE across the intermembrane space to the OE. Here membrane contacts as well as unknown protein partners are likely to be involved. For the FAX protein itself, the transport mode and potential FA/lipid binding domains have to be specified in the future. Further, it has to be clarified if LACS9 at the OE acts together with LACS1, 4, 8 at the ER and if the latter are localized to the outer or inner leaflet of the ER membrane. Again membrane contacts between plastids and the ER most likely are involved in acyl-CoAtransfer to the ER, however also acyl-CoA binding proteins (ACBPs see [10]) might play a role. Finally, the substrate for ABCA9 - free FAs or acyl-CoA — has to be identified. Here somewhat more advanced studies on the peroxisomal FA-importer ABCD1 (see [58]) might serve as a blueprint.

The nature of the lipid imported from the ER to plastids is discussed to be either PA, PC or DAG (see [8]) and the specific transport substrate for the TGD complex is yet unclear. However, it might also 
be possible that different lipids are transferred in parallel or in adaptation to the respective metabolic requirements. Here, the recently discovered co-evolution of a domain in the TGD1 permease protein represents a nice first approach [39*3. While the general transport mechanism of an ABC transporter is obvious, it will be challenging to describe the exact mechanism of lipid transport by the $\beta$-barrel protein TGD4. Most likely TGD4 forms a homodimer, however, if this builds a waterfilled pore as described for 'classical' $\beta$-barrel channels still has to be elucidated. For the postulated transfer of PC from ER to plastids again precise membrane localization of LACS4 is required (see above). Further, it is necessary to clarify how ALIS5 can target ALA10 to the plastid surface, if ALIS5 might be dually localized to ER and plastids and what are the interaction partners in plastids (see Figure 2).

For mitochondrial lipid transport and interaction with other organelles still very little is known in plants (see [44]). Thus, future research should be directed to identify proteins, which mediate organelle contacts as well as lipid traffic. The well known processes in yeast here might serve as a blueprint, however, plant specific metabolic requirements have to be considered. A good system to work on is represented by lipid transfer between plastids and mitochondria under phosphate starvation. However, with At-MIC60 so far only one protein has been identified that is directly mediating transport in mitochondria and chloroplast partners so far are completely unknown (Figure 3).

\section{Acknowledgements}

We first apologize to researchers whose contribution to lipid transporthas not been directly cited in this review due to space limitations. Research on plastid fatty acid export is funded by the DFG grant PH73/7-1 to K.P. Y. L.-B. acknowledges funding from the Agence Nationale de la Recherche (grant MUSCA ANR-13-JSV5-0005). The work of Y.L. was supported by the Advanced Biomass R\&D Center (ABC) of Global Frontier Project funded by the Ministry of Science and ICT (ABC-2015M3A6A2065746).

\section{References}

1 T.H.T. Do, E. Martinoia, Y. Lee Functions of $A B C$ transporters in plant growth and development Curr Opin Plant Biol, 41 (2017), pp. 32-38

\section{$\underline{2}$ E.R. Moellering, C. Benning Galactoglycerolipid metabolism under stress: a time for remodeling} Trends Plant Sci, 16 (2011), pp. 98-107

$\underline{3}$ M.A. Troncoso-Ponce, X. Cao, Z. Yang, J.B. Ohlrogge Lipid turnover during senescence Plant Sci, 205206 (2013), pp. 13-19

4 Y. Nakamura Phosphate starvation and membrane lipid remodeling in seed plants Prog Lipid Res, 52 (2013), pp. 43-50

$\underline{5}$ Y. LiBeisson, B. Shorrosh, F. Beisson, M.X. Andersson, V. Arondel, P.D. Bates, S. Baud, D. Bird,

A. Debono, T.P. Durrett, et al. Acyl-lipid metabolism Arabidopsis Book, 11 (2013), p. e0161 
6 S. Manan, B. Chen, G. She, X. Wan, J. Zhao Transport and transcriptional regulation of oil production in plants Crit Rev Biotechnol, 37 (2017), pp. 641-655

1 A.K. Hurlock, R.L. Roston, K. Wang, C. Benning Lipid trafficking in plant cells Traffic, 15 (2014), pp. 915-932

$\underline{8}$ M.A. Block, J. Jouhet Lipid trafficking at endoplasmic reticulum-chloroplast membrane contact sites Curr Opin Cell Biol, 35 (2015), pp. 21-29

9 P.D. Bates Understanding the control of acyl flux through the lipid metabolic network of plant oil biosynthesis Biochim Biophys Acta, 1861 (2016), pp. 1214-1225

10 Z.Y. Du, T. Arias, W. Meng, M.L. Chye Plant acyl-CoA-binding proteins: an emerging family involved in plant development and stress responses Prog Lipid Res, 63 (2016), pp. 165-181

11 T.A. Salminen, K. Blomqvist, J. Edqvist Lipid transfer proteins: classification, nomenclature, structure, and function Planta, 244 (2016), pp. 971-997

12 M.A. Troncoso-Ponce, K. Nikovics, C. Marchive, L. Lepiniec, S. Baud New insights on the organization and regulation of the fatty acid biosynthetic network in the model higher plant Arabidopsis thaliana Biochimie (2015)

13 C. Somerville, J. Browse Plant lipids: metabolism, mutants, and membranes Science, 252 (1991), pp. $80-87$

14 J. Browse, N. Warwick, C.R. Somerville, C.R. Slack Fluxes through the prokaryotic and eukaryotic pathways of lipid-synthesis in the 16-3 plant Arabidopsis thaliana Biochem J, 235 (1986), pp. 25-31

15 Z. Wang, C. Benning Chloroplast lipid synthesis and lipid trafficking through ER-plastid membrane contact sites Biochem Soc Trans, 40 (2012), pp. 457-463

$\underline{16}$

T. Furumoto, T. Yamaguchi, Y. Ohshima-Ichie, M. Nakamura, Y. TsuchidaIwata, M. Shimamura, J. Ohnishi, S. Hata, U. Gowik, P. Westhoff, et al. A plastidial sodium-dependent pyruvate transporter Nature, 476 (2011), pp. 472-475

17 E.J. Lee, M. Oh, J.U. Hwang, Y. Li-Beisson, I. Nishida, Y. Lee Seed-specific overexpression of the pyruvate transporter BASS2 increases oil content in Arabidopsis seeds Front Plant Sci, 8 (2017), p. 194 In this research, the authors could significantly increase seed TAG content by seed-specific over-expression of a plastid envelope pyruvate transporter. Increased activity of this transporter fuels plastid fatty acid synthesis with C3 carbon units from the cytosol. Thus, the authors show that manipulation of transporter gene levels provides a feasible biotechnological approach to increase seed oils in higher plants.

18 K. Wang, J.E. Froehlich, A. Zienkiewicz, H.L. Hersh, C. Benning A plastid phosphatidylglycerol lipase contributes to the export of acyl groups from plastids for seed oil biosynthesis Plant Cell, 29 (2017), pp. 1678-1696 
Wang et al. in this study nicely demonstrate activity and physiological function of a so far uncharacterized chloroplast specific lipid-degrading enzyme, that is, the phospholipase PLIP1. It is shown that PLIP1 hydrolyzes polyunsaturated acyl groups from a unique plastid intrinsic phophatidylglycerol. Thereby PLIP1 contributes to seed oil biosynthesis and in consequence seed germination. The authors speculate that by releasing free fatty acids in plastids, PLIP1 activity might contribute to plastid fatty acid export.

19 A.J. Koo, J.B. Ohlrogge, M. Pollard On the export of fatty acids from the chloroplast J Biol Chem, 279 (2004), pp. 16101-16110

20 S. Von Berlepsch, H.H. Kunz, S. Brodesser, P. Fink, K. Marin, U.I. Flügge, M.Gierth The acyl-acyl carrier protein synthetase from Synechocystis sp PCC 6803 mediates fatty acid import Plant Physiol, 159 (2012), p. 606

21 N. Li, I.L. Gügel, P. Giavalisco, V. Zeisler, L. Schreiber, J. Soll, K. Philippar FAX1, a novel membrane protein mediating plastid fatty acid export PLoS Biol, 13 (2015), p. e1002053

This work for the first time identifies a membrane-intrinsic transport protein involved in fatty acid export from plastids. Loss of function of the FAX1 protein affects plant biomass, lipid homeostasis as well as ketone wax composition. FAX1 over-expressing lines show an opposite phenotype. The strong defect in the assembly of lipophilic material in outer cell wall of fax 1 knockout pollen grains leads to male sterility. In yeast, Arabidopsis FAX1 could complement for fatty acid transport. Since in vertebrates, FAX1 relatives are structurally related, mitochondrial membrane proteins of so-far unknown function, this protein family might represent a powerful tool not only to increase lipid/biofuel production in plants but also to explore novel FA/lipid transport systems in eukaryotic organelles.

$22 \mathrm{~N}$. Li, C. Xu, Y. Li-Beisson, K. Philippar Fatty acid and lipid transport in plant cells Trends Plant Sci, 21 (2016), pp. 145-158

$\underline{23} \mathrm{~J}$. Andrews, K. Keegstra Acyl-CoA synthetase is located in the outer-membrane and acyl-CoA thioesterase in the inner membrane of pea chloroplast envelopes Plant Physiol, 72 (1983), pp. 735-740

24 J.A. Schnurr, J.M. Shockey, G.J. de Boer, J.A. Browse Fatty acid export from the chloroplast. Molecular characterization of a major plastidial acyl-coenzyme A synthetase from Arabidopsis Plant Physiol, 129 (2002), pp. 1700-1709

25 D. Jessen, C. Roth, M. Wiermer, M. Fulda Two activities of long-chain acyl-coenzyme A synthetase are involved in lipid trafficking between the endoplasmic reticulum and the plastid in ArabidopsisPlant Physiol, 167 (2015), pp. 351-366

This report provides an in-depth phenotypical analysis of single and double knockout mutants for the longchain acyl-CoA synthetases LACS4 and LACS9. While LACS4 is localized in the endoplasmic reticulum, LACS9 sits at the cytosolic face of the plastid outer envelope membrane. The differential lipid distribution in lacs4/lacs9 double mutants suggests an overlapping function of both enzymes in lipid trafficking from the ER to plastids. A model for this transfer via the so-called Lands cycle at ER plastid contact sites is discussed. 
26 L.F. Zhao, V. Katavic, F.L. Li, G.W. Haughn, L. Kuns tinsertional mutant analysis reveals that longchain acyl-CoA synthetase 1 (LACS1), but not LACS8, functionally overlaps with LACS9 in Arabidopsis seed oil biosynthesis Plant J, 64 (2010), pp. 1048-1058

$\underline{27}$ F.L. Theodoulou, I.D. Kerr ABC transporter research: going strong $\mathbf{4 0}$ years on Biochem Soc Trans, 43 (2015), pp. 1033-1040

28 J.U. Hwang, W.Y. Song, D. Hong, D. Ko, Y. Yamaoka, S. Jang, S. Yim, E. Lee, D.Khare, K. Kim, et al. Plant $A B C$ transporters enable many unique aspects of a terrestrial plant's lifestyle Mol Plant, 9 (2016), pp. 338-355

29 S. Kim, Y. Yamaoka, H. Ono, H. Kim, D. Shim, M. Maeshima, E. Martinoia, E.B.Cahoon, I. Nishida, Y. Lee AtABCA9 transporter supplies fatty acids for lipid synthesis to the endoplasmic reticulum Proc Natl Acad Sci USA, 110 (2013), pp. 773-778

30 C.C. Xu, J. Fan, J.E. Froehlich, K. Awai, C. Benning Mutation of the TGD1 chloroplast envelope protein affects phosphatidate metabolism in Arabidopsis Plant Cell, 17 (2005), pp. 3094-3110

31 C.C. Xu, J.L. Fan, W. Riekhof, J.E. Froehlich, C. Benning A permease-like protein involved in ER to thylakoid lipid transfer in Arabidopsis EMBO J, 22 (2003), pp. 2370-2379

32 K. Awai, C.C. Xu, B. Tamot, C. Benning A phosphatidic acid-binding protein of the chloroplast inner envelope membrane involved in lipid trafficking Proc Natl Acad Sci U S A, 103 (2006), pp. 10817-10822

33 B.B. Lu, C.C. Xu, K. Awai, A.D. Jones, C. Benning A small ATPase protein of Arabidopsis, TGD3, involved in chloroplast lipid import J Biol Chem, 282 (2007), pp. 35945-35953

34 Z.Wang, C.C. XU, C. Benning TGD4 involved in endoplasmic reticulum-to-chloroplast lipid trafficking is a phosphatidic acid binding protein Plant $\mathrm{J}, 70$ (2012), pp. 614-623

35 Z. Wang, N.S. Anderson, C. Benning The phosphatidic acid binding site of the Arabidopsis trigalactosyldiacylglycerol 4 (TGD4) protein required for lipid import into chloroplasts

J Biol Chem, 288 (2013), pp. 4763-4771

36 Fan, Z. Zhai, C. Yan, C. Xu Arabidopsis TRIGALACTOSYLDIACYLGLYCEROL5 interacts with TGD1, TGD2, and TGD4 to facilitate lipid transfer from the endoplasmic reticulum to plastids

Plant Cell, 27 (2015), pp. 2941-2955

In this research, the authors find an answer to the question how lipids during import from the ER to plastids can transit across the intermembrane space between plastid outer and inner envelope membranes. By a forward genetic screen, they identified the protein TGD5, that upon mutation in Arabidopsisdisplays similar phenotypes to mutants of TGD4 (lipid import across the outer envelope) as well as of TGD1, TGD2, TGD3 ( $A B C$ transporter complex for lipid transport across the inner envelope). TGD5 is a small, gylcine-rich protein that can physically interact with TGD4 and TGD1-3 and thereby most likely bridges the intermembrane space during plastid lipid import. 
37 E. Dubots, M. Audry, Y. Yamaryo, O. Bastien, H. Ohta, C. Breton, E. Marechal, M.A. Block Activation of the chloroplast monogalactosyldiacylglycerol synthase MGD1 by phosphatidic acid and phosphatidylglycerol J Biol Chem, 285 (2010), pp. 6003-6011

38 A.A. Kelly, B. Kalisch, G. Holzl, S. Schulze, J. Thiele, M. Melzer, R.L. Roston, C.Benning, P. Dörmann Synthesis and transfer of galactolipids in the chloroplast envelope membranes of Arabidopsis thaliana Proc Natl Acad Sci U S A, 113 (2016), pp. 10714-10719

These findings provide molecular insight into intermembrane lipid transfer during the galactolipid synthesis between the two envelope membranes in chloroplasts. The authors nicely show that the $\mathrm{N}$-terminal region of the digalactosyl-diacylglcerol synthase DGD1 at the outer envelope membrane is able to bind phosphatidic acid (PA) and mediate PA-dependent membrane fusion in vitro. Thereby, the N-terminus of DGD1 is proposed to mediate selective channeling of lipid precursors as well as galactolipid translocation between envelopes.

39 Y. Yang, A. Zienkiewicz, A. Lavell, C. Benning Co-evolution of domain interactions in the chloroplast TGD1, 2, 3 lipid transfer complex specific to brassicaceae and poaceae plants Plant Cell (2017)

Here, the authors explored whether the TGD1-3 ABC transporter complex for lipid import into plastids has adapted in its function regarding the lipid metabolism in 16:3 and 18:3 plants (plastid lipids mainly from the prokaryotic and eukaryotic pathways, respectively). They identified the TGD1 orthologue of the 18:3 grass Brachypodium, showed that it is involved in chloroplast lipid import and compared this protein with the 16:3 TGD1 from Arabidopsis. By heterologous complementation analysis in the Arabidopsis tgd1-1 mutant background and co-immunoprecipitation assays, they nicely demonstrate that a 27 amino acid loop of TGD1 that interacts with TGD2 has specifically adapted to the metabolic requirements of either $16: 3$ or $18: 3$ plant species, indicating co-evolution of lipid transport proteins and metabolism.

$\underline{40}$ J. Warakanont, C.H. Tsai, E.J. Michel, G.R. Murphy 3rd, P.Y. Hsueh, R.L. Roston, B.B. Sears, C. Benning Chloroplast lipid transfer processes in Chlamydomonas reinhardtiñnvolving TRIGALACTOSYL DIACYLGLYCEROL 2 (TGD2) orthologue Plant J, 84 (2015), pp. 1005-1020

\section{C. Botella, J. Jouhet, M.A. Block Importance of phosphatidylcholine on the chloroplast surface} Prog Lipid Res, 65 (2017), pp. 12-23

42 C. Botella, E. Sautron, L. Boudiere, M. Michaud, E. Dubots, Y. YamaryoBotte, C.Albrieux, E. Marechal, M.A. Block, J. Jouhet ALA10, a phospholipid flippase, controls FAD2/FAD3 desaturation of phosphatidylcholine in the ER and affects chloroplast lipid composition in Arabidopsis thaliana Plant Physiol, 170 (2016), pp. 1300-1314

This report describes the interesting finding that the ER-resident P4-type ATPase ALA10, depending on the interaction partner - ALIS1 or ALIS5 - can be recruited to either near the plasmamembrane or plastid. Protein interactions are monitored by fluorescence tags and also show interaction of ALA10 with the fatty acid desaturase FAD2 in the ER. Upon over-expression, ALA10 enhances leaf growth and impacts plastid lipid composition, that is, phosphatidylcholine (PC) and monogalaytosyl-diacylglycerol. The authors discuss that ALA10 when interacting with ALIS5 is involved in PC transfer from the ER to plastids.

43 E. Marechal, O. Bastien Modeling of regulatory loops controlling galactolipid biosynthesis in the inner envelope membrane of chloroplasts J Theor Biol, 361 (2014), pp. 1-13 
45 H. Wada, D. Shintani, J. Ohlrogge Why do mitochondria synthesize fatty acids? Evidence for involvement in lipoic acid production Proc Natl Acad Sci U S A, 94 (1997), pp. 1591-1596

46 X. Guan, H. Chen, A. Abramson, H. Man, J. Wu, O. Yu, B.J. Nikolau A phosphopantetheinyl transferase that is essential for mitochondrial fatty acid biosynthesis

Plant J, 84 (2015), pp. 718-732

In this research, the authors characterize the mitochondrial phosphopantetheinyl transferase (mPPT) that activates the acyl carrier protein of mitochondrial fatty acid synthase. Activity of the purified, recombinant enzyme is shown in vitro and in planta function is demonstrated by mutation in Arabidopsis. Here, the loss of mPPT is embryo lethal, while RNAi lines show reduced lipoylation of mitochondrial proteins and typical phenotypes associated with deficiency in photorespiration. Since fatty acid synthesis in mitochondria provides lipoic acid for lipoylation of, for example, enzymes involved in photorespiration, the study demonstrates a direct link between fatty acid synthesis and modulation of essential plant metabolic pathways.

47 X. Guan, B.J. Nikolau AAE13 encodes a dual-localized malonyl-CoA synthetase that is crucial for mitochondrial fatty acid biosynthesis Plant J, 85 (2016), pp. 581-593

Since only little is known about fatty acid synthesis in plant mitochondria, this study provides interesting new insights. The authors could show that due to alternative splicing, the Arabidopsis gene AAE13 encodes for a malonyl-CoA synthetase that dually localizes to the cytosol and mitochondria, respectively. The AAE13 activity in mitochondria is crucial for plant growth as demonstrated by complementation of the respective knockout mutant. Since malonyl-CoA is a substrate for fatty acid synthesis, which in mitochondria produces lipoic acid for lipoylation of enzymes, that in turn regulates metabolic processes like photorespiration, mitochondrial AAE13 represents a vital enzyme required for this process.

48 T. Tatsuta, M. Scharwey, T. Langer Mitochondrial lipid trafficking Trends Cell Biol, 24 (2014), pp. 44-52

49 O. Duncan, N.L. Taylor, C. Carrie, H. Eubel, S. Kubiszewski-Jakubiak, B. Zhang, R. Narsai, A.H. Millar, J. Whelan Multiple lines of evidence localize signaling, morphology, and lipid biosynthesis machinery to the mitochondrial outer membrane of Arabidopsis Plant Physiol, 157 (2011), pp. 1093-1113

$\underline{50}$ S.J. Mueller, D. Lang, S.N. Hoernstein, E.G. Lang, C. Schuessele, A. Schmidt, M. Fluck, D. Leisibach, C. Niegl, A.D. Zimmer, et al. Quantitative analysis of the mitochondrial and plastid proteomes of the moss Physcomitrella patens reveals protein macrocompartmentation and microcompartmentation Plant Physiol, 164 (2014), pp. 2081-2095

51 S.J. Mueller, R. Reski Mitochondrial dynamics and the ER: the plant perspective Front Cell Dev Biol, 3 (2015), p. 78

52 H. Härtel, P. Dörmann, C. Benning DGD1-independent biosynthesis of extraplastidic galactolipids after phosphate deprivation in Arabidopsis Proc Natl Acad Sci U S A, 97 (2000), pp. 10649-10654

53 J. Jouhet, E. Marechal, B. Baldan, R. Bligny, J. Joyard, M.A. Block Phosphate deprivation induces transfer of DGDG galactolipid from chloroplast to mitochondria J Cell Biol, 167 (2004), pp. 863-874

54 M. Michaud, V. Gros, M. Tardif, S. Brugiere, M. Ferro, W.A. Prinz, A. Toulmay, J.Mathur, M. Wozny, 
D. Falconet, et al.AtMic60 is involved in plant mitochondria lipid trafficking and is part of a large complex Curr Biol, 26 (2016), pp. 627-639

By an in depth biochemical analysis of purified native mitochondrial membrane complexes under phosphate sufficient and limiting conditions, the authors were able to identify Mic60 in the inner mitochondrial membrane. It is shown that AtMic60 is involved in export of phosphatidyl-ethanolamine and import of digalctosyldiacylglycerol during phosphate limitation. Whereas during phosphate starvation it is long known that phopholipids in plant organellar membranes are substituted with membrane-forming galactolipids from plastids, the proteins involved in this lipid transfer between organelles so far were unknown. The identification of the Arabidopsis Mic60 protein therefore represents a breakthrough in this field.

$\underline{55}$ R. Schwacke, A. Schneider, E. van derGraaff, K. Fischer, E. Catoni, M.Desimone, W.B. Frommer, U.I. Flugge, R. Kunze ARAMEMNON, a novel database for Arabidopsis integral membrane proteins Plant Physiol, 131 (2003), pp. 16-26

$\underline{56}$ F.K.H. Breuers, A. Brautigam, A.P.M. Weber The plastid outer envelope - a highly dynamic interface between plastid and cytoplasm Front Plant Sci (2011), p. 2

$\underline{57}$ C.D. De Marcos Lousa, C.W.T. van Roermund, V.L.G. Postis, D. Dietrich, I.D.Kerr, R.J.A. Wanders,

S.A. Baldwin, A. Baker, F.L. Theodoulou Intrinsic acyl-CoA thioesterase activity of a peroxisomal ATP binding cassette transporter is required for transport and metabolism of fatty acids

Proc Natl Acad Sci U S A, 110 (2013), pp. 1279-1284

58 A. Baker, D.J. Carrier, T. Schaedler, H.R. Waterham, C.W. van Roermund, F.L.Theodoulou Peroxisomal ABC transporters: functions and mechanism Biochem Soc Trans, 43 (2015), pp. 959-965

59 R.L. Roston, J. Gao, M.W. Murcha, J. Whelan, C. Benning TGD1, -2, and -3 proteins involved in lipid trafficking form ATP-binding cassette $(A B C)$ transporter with multiple substrate-binding proteins J Biol Chem, 287 (2012), pp. 21406-21415 